J. Austral. Math. Soc. 19 (Series A) (1975), 297-305.

\title{
REMARKS ON A CLASS OF 2-GENERATOR GROUPS OF DEFICIENCY ZERO
}

\author{
C. M. CAMPBELL and E. F. ROBERTSON
}

(Received 27 April 1973)

Communicated by G. E. Wall

\section{Introduction}

Let $G$ be a finitely presented group. A finite presentation $\mathscr{P}$ of $G$ is said to have deficiency $m-n$ if it defines $G$ with $m$ generators and $n$ relations. The deficiency of $G$ is the maximum of the deficiencies of all the finite presentations $\mathscr{P}$ of $G$. If $G$ is finite the deficiency of $G$ is less than or equal to zero. The only finite two generator groups of deficiency zero that are known are certain metacyclic groups given by Wamsley (1970), a class of nilpotent groups given by Macdonald in (1962) and a class of groups given by Wamsley (1972).

In this paper we consider a class of two generator groups of deficiency zero. Define the group $\mathbf{G}(m, n)$, where $m, n$ are non-zero integers, by

$$
\mathbf{G}(m, n)=\left\langle a, b \mid\left[a^{m}, b^{-1}\right]=a^{-1} b^{n} a,\left[b^{m}, a^{-1}\right]=b^{-1} a^{n} b\right\rangle .
$$

The groups $\mathbf{G}(1, n)$ are metacyclic groups of order $n^{3}$ and exponent $n^{2}$. We investigate the groups $\mathbf{G}(2, n)$ for $-3 \leqq n \leqq 5$ showing that $\mathbf{G}(2,-3)$ is a group of order $2^{8} \cdot 3^{3}$ and that $\mathbf{G}(2,3)$ is a group of order $2^{15} \cdot 3^{3}$. These two groups are neither metabelian nor nilpotent nor are they isomorphic to any of the groups in the classes defined in Macdonald (1962), and Wamsley (1970), Wamsley (1972). We also show that $\mathbf{G}(3,1)$ is isomorphic to $S L(2,5)$. This answers a question posed in Campbell (1969) as to whether the groups $\mathbf{G}(m, 1)$ are all trivial.

The main tools used in this investigation are the Todd-Coxeter coset enumeration algorithm, see for example Coxeter and Moser (1972), and the modification to the algorithm described in Benson and Mendelsohn (1966). We would like to take this opportunity to thank Dr. M. J. Beetham for allowing us to use his coset enumeration programme Beetham (unpublished). The machine calculations were carried out on the IBM 360 computer of the University of St. Andrews.

\section{The groups $\mathbf{G}(\mathbf{m}, \mathbf{n})$}

We use the notation $\mathbf{Z}_{n}$ for the cyclic group of order $n$. It is easy to see that, if $\mathbf{G}^{\prime}(m, n)$ is the derived group of $\mathbf{G}(m, n)$, then $\mathbf{G}(m, n) / \mathbf{G}^{\prime}(m, n)$ is isomorphic to $\mathbf{Z}_{n} \times \mathbf{Z}_{n}$ if $n \geqq 1$ and to $\mathbf{Z}_{-n} \times \mathbf{Z}_{-n}$ if $n \leqq 1$. 
Consider $\mathbf{G}(1, n)$ for $n \geqq 1$. This group has the presentation

$$
G=\mathbf{G}(1, n)=\left\langle a, b \mid a b^{-1}=b^{n-1} a, b a^{-1}=a^{n-1} b\right\rangle .
$$

Hence $a^{n-1} b \cdot b^{n-1} a=1$ giving $a^{n}=b^{-n}$. Thus $a^{n} \in Z(G)$, the centre of $G$. Since $G /\left\langle a^{n}\right\rangle \simeq \mathbf{Z}_{n} \times \mathbf{Z}_{n}$ we must have $\left\langle a^{n}\right\rangle=G^{\prime}$. Now

$$
b a b^{-1}=b^{n} a=a^{1-n} .
$$

Hence $b^{n} a b^{-n}=a^{1-n^{2}}$ and since $b^{n} a b^{-n}=a, a^{n^{2}}=1$. Therefore $G$ has order $n^{3}$ and is metacyclic of exponent $n^{2}$. If $n \leqq 1$ a similar argument holds. We have the following theorem.

THEOREM 1. If $n \geqq 1, \mathbf{G}(1, n)$ is a finite metacyclic group of order $n^{3}$. The centre of $\mathbf{G}(1, n)$ is equal to the derived group of $\mathbf{G}(1, n)$ and is cyclic of order $n$. The group $\mathbf{G}(1,-n)$ is isomorphic to $\mathbf{G}(1, n)$.

Let $d=$ h.c.f. $(m, n)$. It is easy to see that $\mathbf{G}(m, n)$ has a homomorphic image isomorphic to $\mathbf{Z}_{d} \star \mathbf{Z}_{d}$, the free product of two copies of $\mathbf{Z}_{d}$. For, if $a^{d}=1, b^{d}=1$ are added to $\mathbf{G}(m, n)$, the relations $\left[a^{m}, b^{-1}\right]=a^{-1} b^{n} a$ and $\left[b^{m}, a^{-1}\right]=b^{-1} a^{n} b$ are then redundant. In the case $m=2$ we can prove a slightly stronger result.

THEOREM 2. $\mathbf{G}(m, n)$ has $\mathbf{Z}_{d} \star \mathbf{Z}_{d}$ as a homomorphic image, where $\boldsymbol{d}=$ h.c.f. $(m, n)$. Therefore $\mathbf{G}(m, n)$ is infinite if $m$ and $n$ are not coprime. In $\mathbf{G}(2, n)$, for $n$ even, the subgroup $\left\langle a^{2}, b^{2}\right\rangle$ is normal. $\mathbf{G}(2, n) \mid\left\langle a^{2}, b^{2}\right\rangle$ is isomorphic to $\mathbf{D}_{\infty}$, the infinite dihedral group.

Proof. Since $\mathbf{D}_{\infty} \simeq \mathbf{Z}_{2} \star \mathbf{Z}_{2}$ we need only prove that $\left\langle a^{2}, b^{2}\right\rangle$ is normal in $\mathbf{G}(2, n)$. Since $n$ is even put $n=2 k$. It is sufficient to prove that $a b^{2} a^{-1} \in\left\langle a^{2}, b^{2}\right\rangle$. But

$$
\begin{aligned}
b^{2} & =a^{-1} b a^{2 k} b a \\
& =a^{-1}\left(b a^{2} b^{-1}\right)^{k} b^{2} a \\
& =a^{-1}\left(a b^{2 k} a\right)^{k} b^{2} a \\
& =\left(b^{2 k} a^{2}\right)^{k-1} b^{2 k}\left(a b^{2} a^{-1}\right) a^{2} .
\end{aligned}
$$

Hence $a b^{2} a^{-1} \in\left\langle a^{2}, b^{2}\right\rangle$ as required.

Next we give a result about $\mathbf{G}(m, n)$ where $m, n$ are coprime.

Theorem 3. Let $m$ and $n$ be coprime. Then $\mathbf{G}(m, n)$ has $\mathbf{G}(1, n)$ as a homomorphic image. In particular $\mathbf{G}(-n, n+1)$ is isomorphic to $\mathbf{G}(1, n+1)$.

Proof. Consider the group

$$
H=\left\langle a, b \mid\left[a^{m}, b^{-1}\right]=a^{-1} b^{n} a,\left[b^{m}, a^{-1}\right]=b^{-1} a^{n} b, a^{n} b^{n}=1\right\rangle .
$$

Clearly $H$ is a homomorphic image of $\mathbf{G}(m, n)$. 
Since $a^{n}=b^{-n}, a^{n}$ and $b^{n}$ are in $Z(H)$, the centre of $H$. Hence

$$
b^{-m} a b^{m} a^{-1}=a^{n} \Rightarrow a b^{m} a^{-1}=b^{m} b^{-n} \text {. }
$$

Thus $a^{n} b^{m} a^{-n}=b^{m} b^{-n^{2}}$ and so $b^{n^{2}}=1$. But

$$
\left\langle a, b \mid a^{n}=b^{n}=1,\left[b^{m}, a^{-1}\right]=1,\left[a^{m}, b^{-1}\right]=1\right\rangle \simeq \mathbf{Z}_{n} \times \mathbf{Z}_{n},
$$

since $m$ is coprime to $n^{2}$ and so $\left[b^{m}, a^{-1}\right]=1$ implies that $a$ and $b$ commute. Therefore $H^{\prime}=Z(H)=\left\langle a^{n}\right\rangle$ and $H$ is isomorphic to $\mathbf{G}(1, n)$.

Now consider

$$
\mathbf{G}(-n, n+1)=\left\langle a, b \mid\left[a^{-n}, b^{-1}\right]=a^{-1} b^{n+1} a,\left[b^{-n}, a^{-1}\right]=b^{-1} a^{n+1} b\right\rangle .
$$

The relation $\left[a^{-n}, b^{-1}\right]=a^{-1} b^{n+1} a$ gives $a^{n+1} b a^{-n} b^{-1}=b^{n+1} a$. Also $b^{n+1} a b^{-n} a^{-1}=a^{n+1} b$ and so $a^{n+1} b a^{-n} b^{-(n+1)} a^{-1}=a^{n+1} b$ giving $a^{-(n+1)}$ $=b^{n+1}$. Therefore by the first part of the theorem $\mathbf{G}(-n, n+1)$ is isomorphic to $\mathbf{G}(1, n+1)$.

Let us now consider the infinite groups $\mathbf{G}(2,2 n)$ for $n \geqq 1$. We have proved that $\left\langle a^{2}, b^{2}\right\rangle$ is normal in $\mathbf{G}(2,2 n)$ with $\mathbf{G}(2,2 n) /\left\langle a^{2}, b^{2}\right\rangle$ isomorphic to $\mathbf{D}_{\infty}$. We now give a result which examines defining relations for $\left\langle a, b^{2}\right\rangle$. This in turn gives information about $\left\langle a^{2}, b^{2}\right\rangle$.

The relations of $\mathbf{G}(2,2 n)$ are

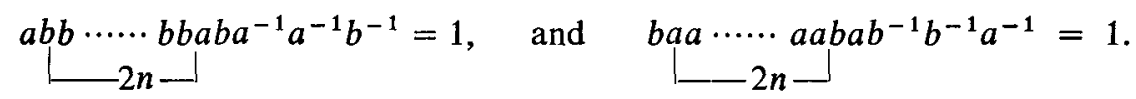

We use the modified Todd-Coxeter algorithm to find a presentation for the subgroup $\left\langle a, b^{2}\right\rangle$. See Beetham and Campbell (to appear) for a proof that the algorithm gives a presentation of the subgroup. We obtain the following table as in Campbell (1969), where $x=a$ and $y=b^{2}$.

$$
\begin{aligned}
& 1 . a=x .1 \\
& 1 . b=1.1 \\
& 2 . b=y .1 \\
& 2 . a=1.3 \\
& 3 . a=x y^{n} x .2 \\
& 3 . b=1.4 \\
& 4 . b=y x^{2 n} .3 \\
& 4 . a=1.5 \\
& 5 . a=x y^{n} x y^{n} .4 \\
& 5 . b=1.6 \\
& 6 . b=y x^{2 n}\left(x y^{n} x\right)^{n} .5 \\
& 6 . a=1.7 \\
& 7 . a=\left(x y^{n} x y^{n}\right)\left(y x^{2 n}\right)^{n} .6
\end{aligned}
$$


It is easy to check, using induction, that if $k$ is odd

$$
\begin{aligned}
& k \cdot a=w(k) \cdot(k-1), \\
& k \cdot b=1 .(k+1),
\end{aligned}
$$

and for $k$ even

$$
\begin{aligned}
& k \cdot b=w(k) \cdot(k-1), \\
& k . a=1 .(k+1),
\end{aligned}
$$

where $w(k)$ is a word in $x$ and $y$ depending on $k$.

Now $w(1)=x, w(2)=y, w(3)=x y^{n} x, w(4)=y x^{2 n}$ and by induction we can show that

$$
w(k)=w(k-2)[w(k-3)]^{n} ; \quad k \geqq 5 .
$$

Each coset gives one relation for the subgroup $\langle x, y\rangle$. Denote by $R(k)=1$ the relation obtained from coset $k$. Then

$$
\begin{aligned}
& R(1)=\left(x y^{n} x\right)^{n} y x y^{-1} x^{-1}, \\
& R(2)=\left(y x^{2 n}\right)^{n} x y^{n} x y x^{-2} y^{-1},
\end{aligned}
$$

and by induction we obtain

$$
R(k)=[w(k+2)]^{n} w(k+1) w(k)[w(k-1)]^{-1}[w(k)]^{-1} ; \quad k \geqq 3 .
$$

Notice that if $n \leqq 1$ precisely the same argument holds. We have proved the following theorem.

Theorem 4. The subgroup $H=\left\langle a, b^{2}\right\rangle$ of $\mathbf{G}(2,2 n)$ has a presentation

$$
H=\langle x, y \mid R(k)=1, k=1,2,3, \cdots\rangle,
$$

where the $R(k)$ are given inductively as above.

\section{Examples}

In this section we examine the groups $G(2, n)$ for $-3 \leqq n \leqq 5$ and also the group $\mathbf{G}(3,1)$.

$\mathbf{G}(2,-3)$ This group has the presentation

$$
\mathbf{G}(2,-3)=\left\langle a, b \mid a b^{2} a^{-1}=b a^{-3} b, b a^{2} b^{-1}=a b^{-3} a\right\rangle \text {. }
$$

Let $H$ be the subgroup of $\mathbf{G}(2,-3)$ generated by $\left[a^{-1}, b^{-1}\right],\left[a^{-1}, b\right],[a, b]$. Clearly $H \leqq \mathbf{G}^{\prime}(2,-3)$ and in fact $|\mathbf{G}(2,-3): H|=18$ so $H$ is a subgroup of index 2 in $\mathbf{G}^{\prime}(2,-3)$. Put $x=\left[a^{-1}, b^{-1}\right], y=\left[a^{-1}, b\right], z=[a, b]$. Using the modification of the Todd-Coxeter coset enumeration algorithm we obtain a presentation for $H$ on the generators $x, y, z$ with the following relations: 


$$
\begin{aligned}
& x^{-2} y z^{-1} y^{-1} x^{2} y^{-1} z y=1 \\
& x^{-1} y z x^{-1} y^{-1} z=1 \\
& y z^{-1} y^{-1} x^{2} z y^{-1} x y^{-1} x=1 \\
& x^{-3} y z y^{-1} x y^{-1} x^{-1} y z^{-1} x^{-1} y^{2}=1 \\
& x^{-2} y z^{-1} y^{-1} x^{2} z^{-1} y^{-1} x z y^{-1} x y z^{-1} y=1, \\
& x y^{-1} z y^{-2} x^{2} y x^{-1} y y^{-1}=1 \\
& y^{2} x z y^{-1} x^{2} y^{-2} x z y x^{-1} y z x y^{-1} z^{-1}=1, \\
& y x^{-1} y z^{-1} x^{-1} y z y^{-1} z^{-1} x^{-1} y^{2} x^{-2} y x^{-1} y z^{-1} x^{-1} y z y^{-2} x y^{-1} x^{-1} y z^{-1} y^{-1} x=1, \\
& y^{-1} z^{2} y^{-1} x^{-1} y z^{-1} x^{-1} y z=1 \\
& x^{-3} y z^{-1} x^{-1} y^{2} x y^{-1} z y^{-1} x^{-1} y=1, \\
& x^{-1} y x^{-1} y z y^{-1} x^{-1} y z^{-2} y x^{-1} y z y^{-2} z y^{-1} z^{-1} y=1, \\
& x^{-1} y x^{-1} y z^{-2} y x^{-1} y^{2} z^{-2} y^{-1} x y^{-1} x^{-2} y=1, \\
& x y^{-2} x z y z^{-1} y^{-1} x z y^{-1} x y^{-1} x^{2} y^{-2} x z y z^{-1} y x^{-1} y z y^{-1}=1, \\
& y^{-1} x y^{-1} x y^{-1} z y^{-1} x^{-1} y z^{-1} y z^{-1} y^{-1} x z y^{-1} x y^{-1} x^{2} z^{-1} y^{-1} x z y^{-1} x y^{-1} x^{2} y^{-2} x z y^{-1} \\
& \quad x y^{-1} x^{2} y^{-2} x z^{2}=1
\end{aligned}
$$

It would not be a too tedious task to produce these defining relations by hand. We however used a programme Wilde (1967) to find the words in the subgroup generators which give the relations between the coset representatives. We wrote a programme to find from these words a presentation for the subgroup $H$. Twenty relations were obtained and the shorter relations used to simplify the longer ones until the programme Beetham (unpublished) could handle the coset enumeration. During the simplifications six of the rrelations were found to be redundant.

The index of $\langle x\rangle$ in $H$ is 32 and $\langle x\rangle$ is not a normal subgroup of $H$. Hence $H$ is not abelian and since $H \leqq \mathbf{G}^{\prime}(2,-3)$ the group $\mathbf{G}(2,-3)$ is not metabelian. In fact $|H|=2^{7} .3$ and so $|\mathbf{G}(2,-3)|=2^{8} .3^{3}$. By Theorem $3 \mathbf{G}(2,-3)$ has a homomorphic image isomorphic to $\mathbf{G}(1,3)$, a group of order 27 , and so $\mathbf{G}(2,-3)$ is an extension of a 2 -group by $\mathbf{G}(1,3)$. Since $|\mathbf{G}(2,-3):\langle a\rangle|=2^{6} .3$ the order of a is 36. Hence $a^{4}$ has order 9 and so $a^{4}$ is contained in a Sylow 3-subgroup $P$. If $\mathbf{G}(2,-3)$ is nilpotent $P$ is normal in $\mathbf{G}(2,-3)$. Let $N$ be the normal closure of $\left\langle a^{4}\right\rangle$ in $\mathbf{G}(2,-3)$. Since $P$ is normal $N \leqq P$ so $\mathbf{G}(2,-3) / N$ has order divisible by $2^{8}$. However $|\mathbf{G}(2,-3) / N|=24$, so $\mathbf{G}(2,-3)$ is not nilpotent.

Next we look at $\mathbf{G}(2,3)$, the structure of which is similar to $\mathbf{G}(2,-3)$.

$\mathbf{G}(2,3)$ This group has the presentation 


$$
\mathbf{G}(2,3)=\left\langle a, b \mid a b^{3} a=b a^{2} b^{-1}, b a^{3} b=a b^{2} a^{-1}\right\rangle .
$$

It is easy to deduce the relations $(a b)^{3}=1$ and $(b a)^{3}=1$ which are of some help in the coset enumerations.

Let $H=\left\langle[a, b],\left[a^{-1}, b^{-1}\right],\left[a^{-1}, b\right]\right\rangle$. Then $H$ is a subgroup of index 18 in $\mathbf{G}(2,3)$ and has index 2 in $\mathbf{G}^{\prime}(2,3)$. With $x=[a, b], y=\left[a^{-1}, b^{-1}\right], z=\left[a^{-1}, b\right]$ we obtain a presentation of $H$ on $x, y, z$ with eight relations

$$
\begin{aligned}
& {\left[x^{2}, y^{2}\right]=1,} \\
& {\left[y^{2}, z^{2}\right]=1,} \\
& z y x^{-1} z^{-1} y x^{-1}=1, \\
& (y x)^{2} z^{-2}=1, \\
& y^{-3} z x^{-1} z^{-1} x^{2} y^{2} z^{-1} x^{2} y^{-1} z x^{-1}=1, \\
& y^{2} z^{-1} x^{2} y^{-1} z x^{2} y^{2} z^{-1} x^{2} y z x^{2}=1, \\
& z^{-1} y^{-2} z x^{-3} y^{-2} z^{-1} y^{-2} z x^{-1} y^{-2}=1, \\
& x^{-1} z^{-1} y^{3} z^{-1} y^{2} x z^{-1} y x^{-2} z=1
\end{aligned}
$$

The same techniques as used for $\mathbf{G}(2,-3)$ were used to find presentations of subgroups of $\mathbf{G}(2,3)$.

The subgroup $\left\langle x^{2}, y^{2}, z^{2}\right\rangle$ is abelian. For, from (3) $\left[z^{2}, y x^{-1}\right]=1$, from (4) $\left[z^{2}, y x\right]=1$ and so $\left[z^{2}, x^{2}\right]=1$. The relations of $H$ are sufficiently complicated to make it very difficult to find a presentation of a subgroup $L$ of $H$ of index greater than two by the modified Todd-Coxeter algorithm. However if the subgroup of $H$ is known to be abelian the simplification is substantial and so it might be reasonable to try to find a presentation for $\left\langle x^{2}, y^{2}, z^{2}\right\rangle$. The index $\left|H:\left\langle x^{2}, y^{2}, z^{2}\right\rangle\right|$ is 256 which the programme Wilde (1967) cannot handle. We reduce the problem by finding a subgroup $K$ of index 2 in $H$ and a larger abelian subgroup than $\left\langle x^{2}, y^{2}, z^{2}\right\rangle$.

Let $A=\left\langle x^{2}, y^{2}, z^{2}, y x^{2} y, y z^{2} y\right\rangle$. Then $A$ is abelian. This can be checked using the relations (1) to (4). We know $x^{2}, y^{2}$ and $z^{2}$ commute. To show $\left[x^{2}, y x^{2} y\right]$ $=1$ use (4) to obtain $\left[x^{2}, y x y x\right]=1$. Since $x^{2}$ commutes with $y x y^{-1},\left[x^{2}, y x^{2} y^{-1}\right]$ $=1$ and so $x^{2}$ commutes with $y x^{2} y$ as required. Next we show that $\left[z^{2}, y x^{2} y\right]=1$. Use (3) to obtain $\left[z^{2}, y x^{-1}\right]=1$. But $\left[z^{2}, y x^{-1}\right]=1$ gives both $\left[z^{2}, x y^{-1}\right]=1$ and $\left[z^{2}, y x\right]=1$ so $\left[z^{2}, y x^{2} y^{-1}\right]=1$ and the result follows. Now $\left[z^{2}, y x^{2} y\right]=1$ implies $\left[x^{2}, y z^{2} y\right]=1$ and it remains to show that $\left[z^{2}, y z^{2} y\right]=1$. But $y z^{2} y$ $=y^{2} x y x y$ by (4) and $z^{2}$ commutes with $x y$ since we have shown above that $\left[z^{2}, x y^{-1}\right]=1$. Hence $A$ is abelian.

Let $K=\left\langle x^{2}, y, z\right\rangle$. Then $H \geqq K \geqq A$ and $|H: K|=2$. With $r=x^{2}, s=y$, $t=z$ we can find a presentation of $K$ on $r, s, t$ with the fourteen relations which may be simplified, using the fact that $A$ is abelian, to the following: 


$$
\begin{aligned}
& {\left[r, t^{2}\right]=1,} \\
& {[r, s r s]=1,} \\
& {\left[r, s t^{2} s\right]=1,} \\
& {\left[r, s^{2}\right]=1,} \\
& {\left[s^{2}, t^{2}\right]=1,} \\
& {\left[r, t s^{2} t\right]=1,} \\
& t^{-2}\left(s r s^{-1} t^{-1} r\right)^{2}=1, \\
& s t r s^{2} t^{-1} r s^{-1} t r s^{2} t^{-1} r=1, \\
& s^{-3} t^{-1} s t s t^{-1} s t=1, \\
& t s r^{-1} t^{-2} s^{-1} r^{-1} t^{-1} s r t^{-2} s^{-1} r^{-1}=1, \\
& t^{-1} s r s t s^{-1} t s r s t^{-1} r s r=1, \\
& t^{-1} s^{-2} t^{-1} r^{-1} s t^{-1} s t^{-2} s t s^{-1} r^{-1}=1, \\
& s^{-1} t s^{-1} t^{2} r^{-1} s^{-2} t^{-1} s^{-1} t s r^{-1} t=1, \\
& s^{3} t^{-1} r s t^{-1} s^{-1} t^{-1} r s t^{-1}=1 .
\end{aligned}
$$

Now $A=\left\langle r, s^{2}, t^{2}, s r s, s t^{2} s\right\rangle$ and $|K: A|=32$. The modified Todd-Coxeter algorithm applied to $A$ as a subgroup of $K$ gives a presentation for the abelian group $A$ from which it can be shown that $|A|=768$ and $r^{12}=\left(s^{2}\right)^{12}=\left(t^{2}\right)^{12}$ $=(s r s)^{12}=\left(s t^{2} s\right)^{4}=1$. This shows that the order of $\mathbf{G}(2,3)$ is $2^{15} \cdot 3^{3}$. Note that $H$ is not abelian since $\langle x\rangle$ is not normal in $H$ and so $\mathbf{G}(2,3)$ is not metabelian. To show $\mathbf{G}(2,3)$ is not nilpotent use the fact that $a b$ is an element of order 3 , so if $\mathbf{G}(2,3)$ is nilpotent $a b$ is contained in the normal Sylow 3-subgroup. This is clearly impossible since $a$ and $b$ have order 72 .

To complete a study of the finite groups $\mathbf{G}(2, n)$ for $-3 \leqq n \leqq 5$ we must examine $\mathbf{G}(2,-1), \mathbf{G}(2,1)$ and $\mathbf{G}(2,5) . \mathbf{G}(2,-1)$ is trivial by Theorem 3 and $\mathbf{G}(2,1)$ is proved to be trivial in Example 2 of Campbell (1969).

$\mathbf{G}(2,5)$ The group $\mathbf{G}(2,5)$ is isomorphic to $\mathbf{G}(1,5)$. To show this we must prove that $a^{5}=b^{-5}$. The subgroup $\langle a\rangle$ is normal in $\mathbf{G}(2,5)$ and $\mathbf{G}(2,5) /\langle a\rangle \simeq \mathbf{Z}_{5}$. Hence $\left\langle a^{5}\right\rangle=\left\langle b^{5}\right\rangle=\mathbf{G}^{\prime}(2,5)$. Since $a^{5}, b^{5} \in Z(\mathbf{G}(2,5))$, the centre of $\mathbf{G}(2,5)$, we have

$$
a^{-2} b a^{2}=b^{6}, \quad b^{-2} a b^{2}=a^{6} .
$$

Then $a^{-2} b^{5} a^{2}=b^{30}$ so $b^{25}=1$. Also $b^{-1} a^{-2} b=b^{5} a^{-2}$ gives $b^{-1} a^{-4} b$ $=b^{10} a^{-4}$, so $b^{-1} a b=b^{10} a$. Thus $b^{-2} a b^{2}=b^{20} a$ and this together with $b^{-2} a b^{2}=a^{6}$ gives $a^{5}=b^{-5}$. 
Next consider the three infinite groups $\mathbf{G}(2,-2), \mathbf{G}(2,2)$ and $\mathbf{G}(2,4)$.

$\mathbf{G}(2,2)$ We prove $\mathbf{G}(2,2)$ is isomorphic to $\mathbf{D}_{\infty}$. This follows from Theorem 2 once we have shown $a^{2}=b^{2}=1$. Now

$$
\mathbf{G}(2,2)=\left\langle a, b \mid a b^{2} a^{-1}=b a^{2} b, b a^{2} b^{-1}=a b^{2} a\right\rangle .
$$

But $a b^{2} a^{-1}=b a^{2} b^{-1} b^{2}=a b^{2} a b^{2}$ and so $a^{-2}=b^{2}$. Then $a b^{2} a^{-1}=b a^{2} b=1$ and so $b^{2}=1$. Since $a^{-2}=b^{2}$ this gives $a^{2}=1$.

$\mathbf{G}(2,-2)$ We show that $\mathbf{G}(2,-2)$ is also isomorphic to $\mathbf{D}_{\infty}$. Use the relations of Theorem 4 to give a presentation for $H=\left\langle a, b^{2}\right\rangle$.

$$
H=\langle x, y \mid R(k)=1, k=1,2,3, \cdots\rangle .
$$

The first four of these relations are

$$
\begin{aligned}
& x^{-2} y x^{-1} y x y^{-1}=1, \\
& x^{2} y^{-1} x y^{-1} x y x^{-2} y^{-1}=1, \\
& y x^{-1} y x^{-1} y x^{-1} y^{-1} x y^{-1} x^{-1} y x^{-1}=1, \\
& x y^{-1} x^{3} y^{-1} x y^{-1} x^{-2} y x y^{-1}=1 .
\end{aligned}
$$

It is straightforward to deduce from these relations that $y=1$ and $x^{2}=1 . H$ is isomorphic to the cyclic group of order 2 since the relations $R(k)=1$ hold in the group $\left\langle x, y \mid y=1, x^{2}=1\right\rangle$ for $k \geqq 5$. This gives $a^{2}=1$ and therefore $b^{2}=1$. Thus $\mathbf{G}(2,-2)$ is isomorphic to the infinite dihedral group by Theorem 2 .

$\mathbf{G}(2,4)$ Again use Theorem $4 . H$ can easily be shown to have only five relations. For, $w(k)=w(k-2)[w(k-3)]^{2}, k \geqq 5$, and

$$
[w(k+2)]^{2} w(k+1) w(k)[w(k-1)]^{-1}[w(k)]^{-1}=1, \quad k \geqq 3 .
$$

Then $w(k+2)=w(k)[w(k-1)]^{2}$ and so $w(k+2) w(k+1) w(k) w(k-1)=1$, $k \geqq 3$. Then $w(k+3)=w(k-1), k \geqq 3$. Hence, for $n=2, H$ has a presentation

$$
H=\langle x, y \mid R(1)=R(2)=R(3)=R(4)=R(5)=1\rangle \text {. }
$$

This presentation simplifies to

$$
H=\left\langle x, y \mid y^{-1} x y=x^{-3}, x y^{2} x^{-1}=y^{-2}, y x^{-1} y^{-1}=x^{-5}\right\rangle,
$$

and so $H$ is a group of order 64 . Hence $\left\langle a^{2}, b^{2}\right\rangle$ is a group of order 32 whose structure is easily exhibited. $\mathbf{G}(2,4)$ is an extension of this group of order 32 by $\mathbf{D}_{\infty}$

$\mathbf{G}(3,1) \quad$ This group has the presentation

$$
\mathbf{G}(3,1)=\left\langle a, b \mid a b^{3}=b^{2} a b a, b a^{3}=a^{2} b a b\right\rangle .
$$

The order of $\mathbf{G}(3,1)$ is 120 . For, using the modified Todd-Coxeter algorithm on the subgroup $\langle a\rangle$ gives $|\mathbf{G}(3,1):\langle a\rangle|=12$ and the presentation for $\langle a\rangle$ obtained is $\left\langle a \mid a^{10}=1\right\rangle$. 
$S L(2,5)$ is generated by the two matrices $A$ and $B$ where

$$
A=\left(\begin{array}{ll}
4 & 0 \\
4 & 4
\end{array}\right), \quad B=\left(\begin{array}{ll}
4 & 3 \\
0 & 4
\end{array}\right) \text {. }
$$

But $A B^{3}=B^{2} A B A$ and $B A^{3}=A^{2} B A B$ and so $S L(2,5)$ is a homomorphic image of $\mathbf{G}(3,1)$. However $|S L(2,5)|=120$ and so $\mathbf{G}(3,1)$ is isomorphic to $S L(2,5)$.

\section{References}

M. J. Beetham (unpublished), 'A programme for the Todd-Coxeter coset enumeration algorithm'. M. J. Beetham and C. M. Campbell (to appear), 'A note on the Todd-Coxeter coset enumeration algorithm'.

C. T. Benson and N. S. Mendelsohn (1966), 'A calculus for a certain class of word problems in groups', J. Combinatorial Theory 1, 202-208.

C. M. Campbell (1969), 'Some examples using coset enumeration', in Computational Problems in Abstract Algebra, edited by J. Leech (Pergamon, Oxford, 1969)

H. S. M. Coxeter and W. O. J. Moser (1972), Generators and Relations for Discrete Groups (Springer, Berlin, 3rd. ed. 1972).

I. D. Macdonald (1962), 'On a class of finitely presented groups', Canad. J. Math. 14, 602-613.

J. W. Wamsley (1970), 'The deficiency of metacyclic groups', Proc. Amer. Math. Soc. 24, 724-726.

J. W. Wamsley (1972) 'A class of two generator two relation finite groups', J. Austral. Math. Soc. $14,38-40$.

N. W. G. Wilde (1967), 'Benson Mendelsohn algorithm for certain word problems in groups. I.B.M. Contributed Program Library, No. 42.0.001.

Mathematical Institute

University of St. Andrews

St. Andrews, Fife

Scotland 